THE AsTrophysical JouRnal, 481:758-763, 1997 June 1

(C) 1997. The American Astronomical Society. All rights reserved. Printed in U.S.A.

\title{
DETECTION OF MULTI-TeV EMISSION FROM MARKARIAN 421
}

\author{
F. Krennrich, ${ }^{1}$ C. W. AKerlof, ${ }^{2}$ J. H. Buckley, ${ }^{3}$ A. M. Burdett, ${ }^{4}$ D. A. Carter-Lewis, ${ }^{1}$ M. F. CAwley, ${ }^{5}$ \\ M. Catanese, ${ }^{1}$ V. Connaughton, ${ }^{6}$ D. J. Fegan, ${ }^{6}$ J. P. Finley,${ }^{7}$ J. A. Gaidos, ${ }^{7}$ \\ R. C. Lamb, ${ }^{1}$ R. Lessard, ${ }^{6}$ J. E. McEnery, ${ }^{6}$ G. Mohanty, ${ }^{1}$ J. Quinn, ${ }^{6}$ \\ A. J. Rodgers, ${ }^{4}$ H. J. Rose, ${ }^{4}$ M. S. Schubnell, ${ }^{2}$ G. H. Sembroski, ${ }^{7}$ \\ T. C. WEEKES, ${ }^{3}$ C. WILSON, ${ }^{7}$ AND J. ZWEERINK ${ }^{1}$ \\ Received 1996 September 24; accepted 1996 December 30
}

\begin{abstract}
Gamma rays with energies exceeding $5 \pm 1.5 \mathrm{TeV}$ have been detected from Markarian 421 using the Whipple Observatory's $10 \mathrm{~m} \gamma$-ray telescope. These observations employ a new technique: the so-called large zenith-angle technique. Because they are taken at large zenith angles, the observations yield high statistics data on the multi-TeV part of the spectrum and are well suited for examining the question of a possible energy cutoff. Observations taken during high states on 1995 June 20, 21, and 28 show no evidence for a spectral break. These results conflict with a previous interpretation of the Markarian 421 energy spectrum in which a cutoff due to $\gamma$-ray absorption in extragalactic space was postulated.

Subject headings: BL Lacertae objects: individual (Markarian 421) — gamma rays: observations
\end{abstract}

\section{INTRODUCTION}

In this paper we present new observations of Mrk 421 by the Whipple Observatory collaboration. The observations employ the atmospheric Cerenkov technique, but with a significant difference from previous observations: the data reported here are taken at large zenith angles. This causes the energy region covered by the observations to shift to significantly higher energies than standard small zenithangle observations. Also, the detector collection area increases and, as a consequence, the large zenith-angle observations have a good sensitivity above $5 \mathrm{TeV}$, in contrast to our previous reported observations (Mohanty et al. 1993). In $\S 2$ we present the results of Monte Carlo simulations that contrast the large zenith-angle technique with the normal atmospheric Cerenkov technique. To confirm the findings of these simulations, we use large zenith-angle observations of the Crab Nebula. In $\S \S 3$ and 4 large zenithangle observations of Mrk 421 are presented and analyzed, with particular emphasis on the highest photon energies. These data also show the short-term variability of Mrk 421, which has been well documented (Kerrick et al. 1995; Gaidos et al. 1996; Buckley et al. 1996). Finally, § 5 contains a summary of the results and a discussion of their implications.

Previous observations of $\mathrm{TeV} \gamma$-ray emission from Markarian 421 (Mrk 421) by Mohanty et al. (1993) have been used by a number of authors to derive constraints on the density of intergalactic starlight. The physical process is $\gamma \gamma \rightarrow e^{+} e^{-}$, the absorption of the high-energy radiation through pair production of optical or near-infrared

\footnotetext{
${ }^{1}$ Department of Physics and Astronomy, Iowa State University, Ames, IA 50011-3160.

${ }^{2}$ Randall Laboratory of Physics, University of Michigan, Ann Arbor, MI 48109-1120.

${ }^{3}$ Fred Lawrence Whipple Observatory, Harvard-Smithsonian Center for Astrophysics, P.O. Box 97, Amado, AZ 85645-0097.

${ }_{4}^{4}$ Department of Physics, University of Leeds, Leeds, LS2 9JT, Yorkshire, England, UK.

${ }^{5}$ Physics Department, St. Patrick's College, Maynooth, County Kildare, Ireland.

${ }^{6}$ Physics Department, University College, Dublin 4, Ireland.

${ }^{7}$ Department of Physics, Purdue University, West Lafayette, IN 47907.
}

photons. By making the most conservative assumption that appreciable absorption was already taking place at the lowest energies covered by the Whipple Observatory measurements $(500 \mathrm{GeV})$, Biller et al. (1995a) derived upper limits on that density. De Jager, Stecker, \& Salamon (1994) derived a measurement (as opposed to an upper limit) for the starlight density by integrating a preliminary Whipple Observatory differential spectrum (Mohanty et al. 1993). De Jager, Stecker, \& Salamon (1994) made the assumption that absorption was taking place external to the object itself. Our new observations do not support the detection of intergalactic absorption.

\section{MONTE CARLO SIMULATIONS: THE LARGE ZENITH-ANGLE TECHNIQUE}

Given their limited duty cycle $(\leq 10 \%)$, imaging atmospheric Cerenkov telescopes are inefficient in detecting $\gamma$-rays in the energy range $1-20 \mathrm{TeV}$ using the conventional observing mode at zenith angles $\leq 35^{\circ}$. The reason is that for telescopes employing cameras with fields of view of less than $3^{\circ}$, the collection area is limited to $\sim 40,000 \mathrm{~m}^{2}$ (Reynolds et al. 1993). At large zenith angles this is no longer true. The possibility of detecting $\mathrm{PeV}\left(10^{15} \mathrm{eV}\right)$ $\gamma$-rays at extremely large zenith angles with Cerenkov telescopes was suggested by Sommers \& Elbert (1987). They pointed out that it might be feasible to detect a $\mathrm{PeV} \gamma$-ray source at large zenith angles. However, the proposed technique at zenith angles of more than $80^{\circ}$ suffers from difficulties arising from a significantly thicker atmosphere. Monte Carlo codes have been tested adequately for angles less than $65^{\circ}$. Therefore we have restricted our approach to the zenith angle range of $0^{\circ}-65^{\circ}$. A report by the CANGAROO collaboration indicated evidence for a detection of a $\gamma$-ray signal at large zenith angles $\left(53^{\circ}-56^{\circ}\right)$ from the Crab Nebula (Tanimori et al. 1994).

The Whipple Observatory's $10 \mathrm{~m}$ telescope has been modeled using the Monte Carlo shower simulation programs KASCADE (Kertzman \& Sembroski 1994) and ISUSIM developed by Mohanty et al. (1996). Here we use the ISUSIM Monte Carlo to calculate the detector response at different zenith angles. The simulation incorporates the main features of the Whipple Observatory's $10 \mathrm{~m}$ 
telescope, including mirror aberrations (Lewis 1990). The estimated trigger threshold is $250 \mathrm{GeV}$ at the zenith.

For this work, Monte Carlo simulations of photoninduced showers striking the atmosphere at different zenith angles have been carried out. Gamma-ray showers at $20^{\circ}$, $45^{\circ}$, and $55^{\circ}$ zenith angle have been generated falling up to $650 \mathrm{~m}$ from the telescope. We have considered a large range for the shower impact radius in order to account for the large collection areas at large zenith angles.

The overall normalization for the conversion of total recorded Cerenkov light to primary $\gamma$-ray energy is difficult to determine (Biller et al. 1995b). Approaches include direct measurement of the response of each component (mirror reflectivity, photomultiplier quantum efficiency, light cone collection efficiency, etc.), use of light rings from local muons (Rose et al. 1995), and Monte Carlo simulations of hadronic background showers (Mohanty et al. 1996). The work here uses results from the direct measurement approach, though the agreement with other methods is within $30 \%$. Uncertainty in this normalization causes a corresponding uncertainty (about 30\%) in the energy scale.

\subsection{Image Analysis}

The Whipple Observatory collaboration uses a standard procedure, Supercuts (Reynolds et al. 1993), to enhance the $\gamma$-ray signal over the hadronic background. The Supercuts analysis employs the image parameters (Hillas 1985) width, length, distance, and alpha (hereafter $\alpha$ ), and potential $\gamma$-ray candidates must fall into a certain region of these image parameters. These cuts have been optimized on observations of the Crab Nebula recorded at zenith angles less than $35^{\circ}$ (Reynolds et al. 1993) and remove more than $99.7 \%$ of background cosmic-ray showers. However, they are not the most efficient selection criteria for $\gamma$-ray events at large zenith angles because those air showers produce narrower Cerenkov images.

The analysis technique developed here for large zenithangle $\gamma$-ray showers follows the same principles as those used for observations taken close to the zenith. We separate $\gamma$-ray showers from hadronic background showers using parameters describing the shape of shower images such as width and length and the distance parameter, which measures the distance of the centroid of an image from the camera center. Those parameters can be used for the large zenith-angle data analysis as well. However, a relative shift of the parameter distributions resulting from the zenithangle dependence needs to be taken into account. Showers at large zenith angles are farther away from the telescope, and the lateral extent at shower maximum seen by an imaging instrument appears to be smaller. To determine the shift of the parameter distributions we rely on simulations of $\gamma$-ray showers passing through the atmosphere at different zenith angles.

Comparison of the width distribution for $\gamma$-ray showers at two different zenith angles $\left(20^{\circ}\right.$ and $\left.55^{\circ}\right)$ is illustrated in Figure 1. As can be readily seen, the width parameter of $\gamma$-ray showers at $55^{\circ}$ zenith angle is, on average, $15 \%$ smaller, suggesting a reduction in the width cut from 0.15 to 0.12 . The average length value is expected to shift toward smaller values when the shower develops farther away from the telescope. However, this is somewhat compensated by the larger average impact parameters of the showers at large zenith angles, since the shower images get more elongated when the shower core is farther away from the tele-

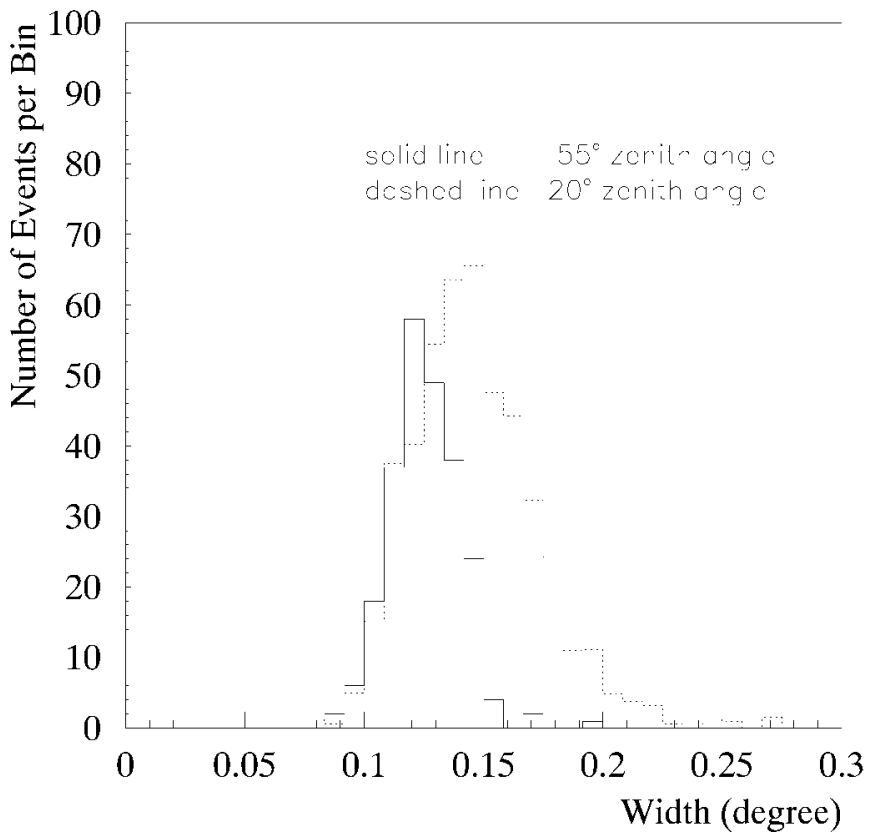

FIG. 1.-Width distribution of $\gamma$-ray showers at $55^{\circ}$ (solid line) and $20^{\circ}$ (dashed line) zenith angles. The events were sampled from a power-law spectrum with a differential index of 2.5. Total light (size) of more than 400 photoelectrons is required. As can be seen from the two distributions, the width values are on average smaller at large zenith angles.

scope. We conclude from Monte Carlo simulations that a good sensitivity for large zenith-angle observations can be reached by using the cuts for different zenith-angle bins as shown in Table 1.

In order to use a similar approach to that in Reynolds et al. (1993), by using experimental data on a known source to optimize the cut criteria for large zenith-angle observations, more statistics are required and could be achieved with more extensive observations.

\subsection{Energy Threshold and Energy Resolution}

As emphasized in $\S 1$, both the energy threshold and the collection area are expected to increase with large zenith angles. We have used Monte Carlo simulations to determine the energy threshold. The total measured light of a shower image provides information about the energy of the primary $\gamma$-ray. For on-axis $\gamma$-ray showers there is a strong correlation between the primary energy and the total light contained in the camera. In order to avoid an artificial loss of this correlation arising from images only partly contained in the camera, an upper distance cut of 0.90 is applied for the following analysis. A lower distance cut of 0.50 has also been applied, since $\gamma$-ray images in the dis-

TABLE 1

IMAGe SELECTION CRITERIA FOR DifFERENT ZENITH ANGLES

\begin{tabular}{cccc}
\hline \hline Zenith Angle & Width Cut & Length Cut & $\alpha$ Cut \\
\hline $0^{\circ}-40^{\circ} \ldots \ldots \ldots$ & 0.150 & 0.26 & $15^{\circ}$ \\
$40^{\circ}-45^{\circ} \ldots \ldots$. & 0.145 & 0.25 & $15^{\circ}$ \\
$45^{\circ}-50^{\circ} \ldots \ldots$. & 0.140 & 0.25 & $15^{\circ}$ \\
$50^{\circ}-52.5 \ldots \ldots$ & 0.135 & 0.25 & $15^{\circ}$ \\
$52.5-55^{\circ} \ldots \ldots$ & 0.130 & 0.25 & $15^{\circ}$ \\
$55^{\circ}-57.5 \ldots \ldots$ & 0.125 & 0.25 & $15^{\circ}$ \\
$57.5-60^{\circ} \ldots \ldots$ & 0.120 & 0.25 & $15^{\circ}$ \\
\hline
\end{tabular}


tance regime less than 0.50 correspond to shower core impact points less than $\sim 50 \mathrm{~m}$ (at $20^{\circ}$ zenith angle) from the telescope, where the light intensity is not well correlated with the primary energy.

Figure 2 shows the energy distribution of $\gamma$-ray primaries (striking the atmosphere at two different zenith angles), for showers that deposit 350-400 photoelectrons in the Whipple Observatory's $10 \mathrm{~m}$ telescope camera. The simulated $\gamma$-ray primaries have been sampled from a differential power law energy distribution of $E^{-2.5}$. According to Figure 2, the average energy increases significantly for the large zenith-angle showers $\left(55^{\circ}\right)$ in comparison to small zenith angles. Using, for example, events with 350-400 photoelectrons ( 1 photoelectron $\approx 1$ digital count, which is the unit given by the analog to digital converter, see Biller et al. 1995b) the average primary energy goes from $380 \mathrm{GeV}$ at $20^{\circ}$ zenith angle up to $800 \mathrm{GeV}$ at $45^{\circ}$ zenith angle and reaches $1.7 \mathrm{TeV}$ for $55^{\circ}$ zenith angle observations. From Figure 2, it can also be seen that the energy resolution $(\Delta E / E)$ does not change significantly for large zenith angles $\left(55^{\circ}\right)$ and is comparable to $20^{\circ}$ zenith-angle observations at the energy range shown. The energy resolution remains at $\sim 40 \%$ using the total detected light for energy estimation (energy resolution defined as $1 \sigma$ ). The accuracy of deriving energy spectra from large zenith-angle observations should be comparable to standard small zenith-angle observations.

It should be noted that extraction of energy spectra is also possible for variable sources, using different zenithangle observations. In Figure 3 we show the correlation between the measured light and the corresponding average energy. The vertical error bars exhibit the statistical error on the mean energy, and the horizontal bars reflect the bin size. As can be seen, data at the different zenith angles provide enough overlap in energy to make it possible to detect systematic flux variations within the time of different zenith-angle observations. Figure 3 also shows that the

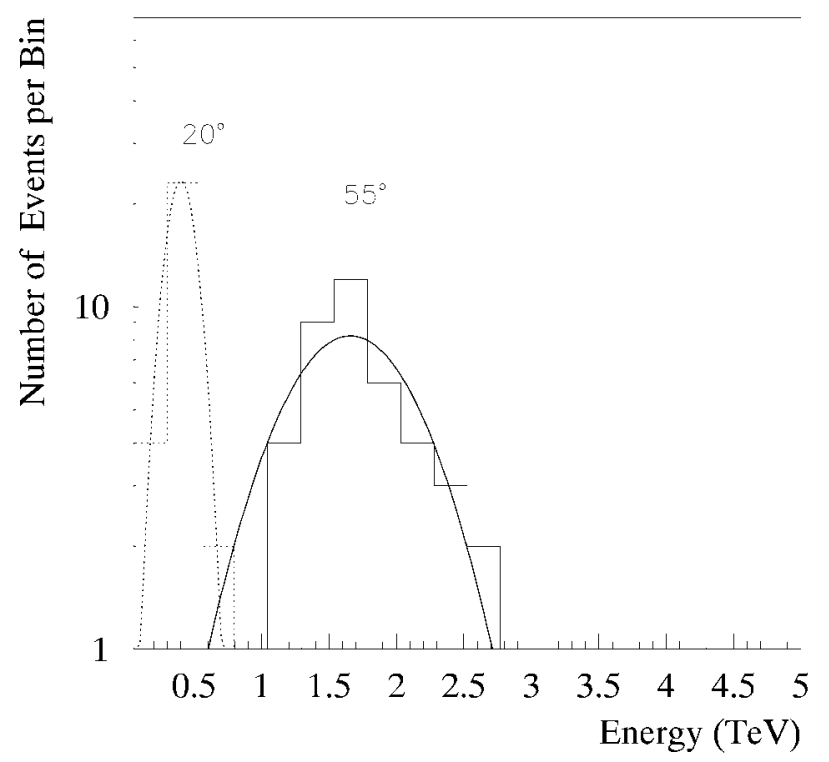

FIG. 2.-Energy distribution for simulated $\gamma$-ray showers at $20^{\circ}$ (dashed line) and at $55^{\circ}$ (solid line) zenith angle (sampled from a power-law spectrum with a differential index of 2.5) with a size of 350-400 digital counts. These distributions reflects the energy distribution corresponding a particular size interval. It can be seen that the average energy at $55^{\circ}$ zenith angle goes up to $1.7 \mathrm{TeV}$, where at $20^{\circ}$ zenith angle the average energy is 380 $\mathrm{GeV}$.

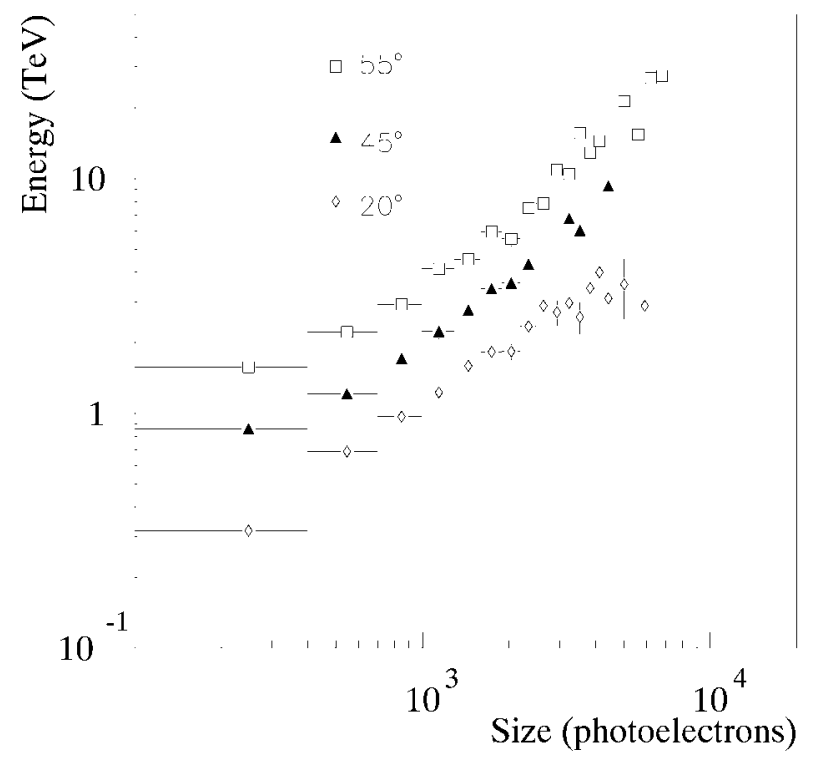

FIG. 3.-Average energy of $\gamma$-ray showers as a function of the total measured light at different zenith angles $\left(20^{\circ}, 45^{\circ}\right.$, and $\left.55^{\circ}\right)$.

dynamic range that can be covered with the Whipple Observatory's $10 \mathrm{~m}$ Cerenkov telescope extends from 300 $\mathrm{GeV}$ up to $20 \mathrm{TeV}$. However, given the nature of the analog to digital converters currently being used, saturation starts to diminish the correlation of the energy with the total light at size levels of around 6000 digital counts. This distorts the energy resolution, particularly at energies above $4 \mathrm{TeV}$ for small zenith-angle observations. At large zenith angles saturation starts to affect the energy resolution at energies $\sim 20 \mathrm{TeV}$.

In the following analysis, the energy threshold is defined so that $90 \%$ of all events that pass the corresponding size cut have energies above that threshold. This definition is more conservative than that normally used but ensures that most of the events really are above a certain energy threshold. The size cut ascribed to a certain energy can approximately be extracted from Figure 3, though the exact value is given more accurately by the definition above. It should be noted that the energy thresholds derived from Monte Carlo simulations rely on the absolute energy calibration of the instrument for which an uncertainty of $30 \%$ is quoted.

\subsection{Collection Area}

The collection area for $\gamma$-ray showers increases with increasing zenith angles, as the region of light production is farther away and consequently the light spreads out over a larger area. To quantify the relation between collection area and zenith angle we have used $\gamma$-ray showers distributed in energy with a power law of $E^{-2.5}$ at $20^{\circ}, 45^{\circ}$, and $55^{\circ}$ zenith angle. The collection area $A_{\text {coll }}$ is defined for $\gamma$-rays from a point source by:

$$
A_{\text {coll }}=2 \pi \int_{0}^{\infty} P_{\gamma}(E, r) r d r,
$$

where $P_{\gamma}(E, r)$ is the detection efficiency for a $\gamma$-ray primary of energy $E$ and impact parameter $r$. Here we compare collection areas for those $\gamma$-ray events that provide a good energy resolution. We have applied a cut on the distance parameter using only events having distance values between 0.50 and 0.90 . A size cut on the minimum required light in 
TABLE 2

COllection Area for Different Zenith ANGLes

\begin{tabular}{lcc}
\hline \hline Zenith Angle & $\begin{array}{c}\text { Effective Collection Area } \\
A_{\text {coll }}\left(\mathrm{m}^{2}\right)\end{array}$ & $A_{\text {coll }} / A_{\text {coll }}\left(20^{\circ}\right)$ \\
\hline $20^{\circ} \ldots \ldots \ldots \ldots$ & $40000 \pm 4000$ & 1.0 \\
$45^{\circ} \ldots \ldots \ldots \ldots$ & $90000 \pm 10000$ & 2.3 \\
$55^{\circ} \ldots \ldots \ldots \ldots$ & $214000 \pm 15000$ & 5.3 \\
\hline
\end{tabular}

the camera of 400 photoelectrons has been applied for this comparison.

The effective collection area, $A_{\text {eff }}$, is obtained by calculating the collection area as a function of the $\gamma$-ray energy $E$. $A_{\text {coll }}(E)$ is a rising function with increasing energy and in general shows a plateau toward higher energies. Its plateau value is chosen here to be the effective collection area $\left(A_{\text {eff }}\right)$. The effective collection area for different zenith angles is the area projected into the plane perpendicular to the source direction.

Table 2 shows that, compared to the value at a zenith angle of $20^{\circ}$, the effective collection area increases by factors of 2.3 and 5.3 at zenith angles of $45^{\circ}$ and $55^{\circ}$, respectively. The given error bars on the effective collection area are purely statistical.

The increase of the energy threshold for the three different zenith angles is similar to the increase in effective collection area. As a consequence, for a source spectrum with an integral index of 1.0, the loss in sensitivity due to the increase in energy threshold is compensated by the increase in effective collection area.

\subsection{Large Zenith-Angle Observations of the Crab Nebula}

The Crab Nebula, which is regarded as the "standard candle" in TeV $\gamma$-ray astronomy (Weekes et al. 1994), serves here as a test beam to perform a consistency check of the predictions of Monte Carlo simulations regarding the energy threshold and collection area at different zenith angles.

During the 1995-1996 observation season, observations of the Crab Nebula were carried out on the same night at two different zenith-angle regions $\left(5^{\circ}-30^{\circ}\right.$, and $\left.55^{\circ}-60^{\circ}\right)$. We can compare the number of excess events for two data sets consisting of 6 oN/OFF measurements each, taken at two different zenith-angle ranges by selecting size cuts that impose the same energy threshold in both data sets. Again, a restriction of the distance parameter to the region between 0.50 and 0.90 has been applied in order to ensure a good energy resolution. The energy threshold has been set to $2 \pm 0.6 \mathrm{TeV}$ for both data sets, and $90 \%$ of all events are expected to be above $2 \mathrm{TeV}$.

From Table 3, it may be seen that the number of excess events at $55^{\circ}-60^{\circ}$ is increased by a factor of $4.2 \pm 1.2$. The relative increase of number of excess events for the $55^{\circ}-60^{\circ}$ data reflects the relative increase in collection area, assuming that the two energy thresholds are indeed the same. This result is in agreement with the relative increase

TABLE 3

NuMBER of Excess Events AT DIFFERENT ZeNith Angles (Crab Nebula)

\begin{tabular}{lcc}
\hline \hline Zenith Angle & Excess Events & $A_{\text {coll }} / A_{\text {coll }}\left(20^{\circ}\right)$ \\
\hline $5^{\circ}-30^{\circ} \ldots \ldots$ & $21 \pm 5$ & 1 \\
$55^{\circ}-60^{\circ} \ldots \ldots$ & $89 \pm 15$ & $4.2 \pm 1.2$ \\
\hline
\end{tabular}

in effective collection area as calculated by Monte Carlo simulations in $\S 2.2$.

\section{DETECTION OF MARKARIAN 421 AT LARGE ZENITH ANGLES}

The large zenith-angle observation technique to measure multi-TeV $\gamma$-ray emission was used to observe Mrk 421 during 1995 June. The data collected with the Whipple Observatory's $10 \mathrm{~m}$ telescope between 1995 June 18 and July 1 are the subject of the analysis to be discussed here. The data consist of 17 oN/OFF pairs and two tracking runs taken at zenith angles between $42^{\circ}$ and $60^{\circ}$. Applying the fine binned large zenith-angle Supercuts of $\S 2$ and a size cut of $\geq 300$ digital counts to the 17 oN/OFF pairs, a positive detection with a significance of $14.3 \sigma\left[\sigma=N_{\text {on }}-N_{\text {off }} /\right.$ $\left(N_{\text {on }}+N_{\text {off }}\right)^{1 / 2}, N_{\text {on }}=$ number of excess events of on source data and $N_{\text {off }}=$ number of excess events of oFF source data] is obtained for the complete data set (Fig. 4). The standard Supercuts analysis results in a detection with a significance of $8.8 \sigma$. The large zenith-angle Supercuts derived above results in an increase in sensitivity by a factor of 1.66 in comparison to the standard Supercuts. This result is the first detection of a source with a significance of more than $5 \sigma$ at large zenith angles, as previously reported by Krennrich et al. (1995).

Since Mrk 421 has been seen as a highly variable source, we have calculated the daily rate variations for 11 nights of observation. To ensure that rate variations present in the data are not affected by different zenith angles, we have chosen two fixed zenith-angle intervals, $45^{\circ}-50^{\circ}$ and $55^{\circ}-$ $60^{\circ}$ to study rate variations. The photon rates for Mrk 421 derived from observations from June 18 until July 1 are shown in Figures $5 a$ and $5 b$. The data show evidence for two outbursts of $\gamma$-ray emission. The burst on June 20 (burst 1) and the burst on June 28 (burst 2) indicate significant flux variations of Mrk 421 at energy thresholds of $800 \mathrm{GeV}$ $\left(45^{\circ}-50^{\circ}\right)$ and $2 \mathrm{TeV}\left(55^{\circ}-60^{\circ}\right)$.

Burst 1 was used to address the possibility of extracting spectral information from large zenith-angle observations. Here we demonstrate the dynamic range that can be covered during a single night of observations. This has become an important scientific issue since day to subday scale variabilities from active galactic nuclei (AGNs) have

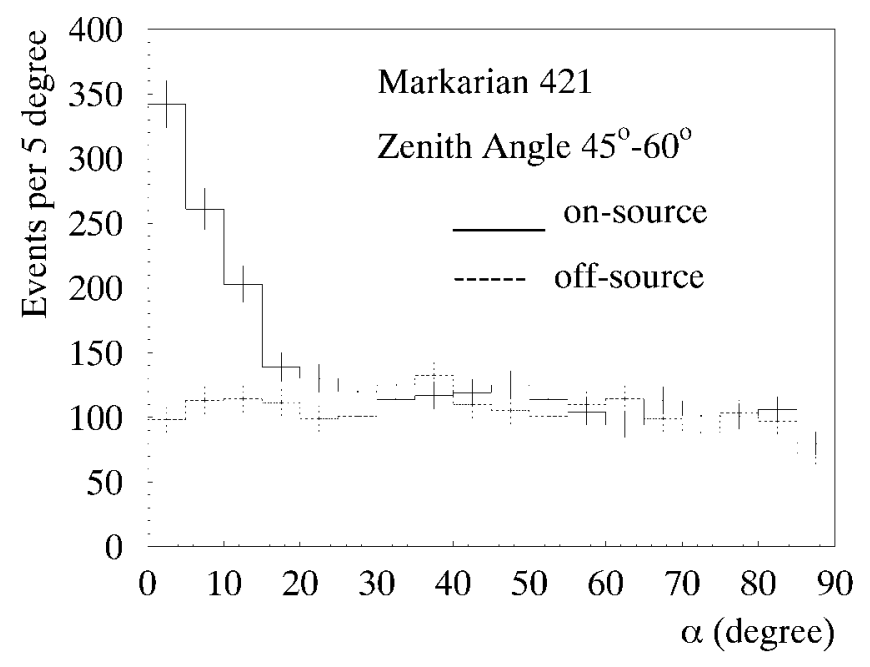

FIG. 4.-The $\alpha$ distribution for the complete data set (June 18 until July 1) of Mrk 421 at large zenith angles. 


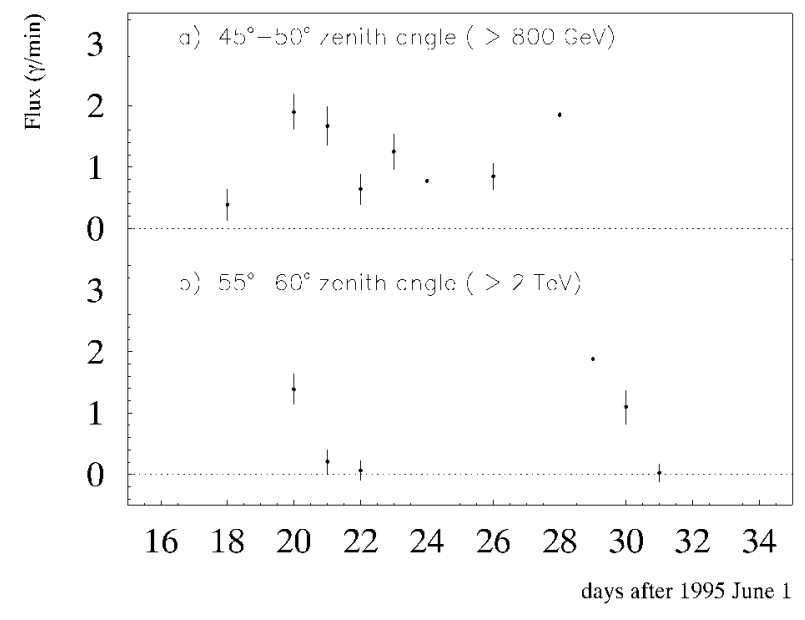

FIG. 5.-The $\gamma$-ray rate for different nights between June 18 and July 1 at two different zenith-angle regions.

been detected in TeV $\gamma$-rays (Gaidos et al. 1996; Quinn et al. 1996). Combining the large zenith-angle technique with data taken in standard observation mode should provide a rather large dynamic energy range. Based on the 4-5 times larger collection area at $55^{\circ}$ zenith angle in comparison with that at $20^{\circ}$, the sensitivity for energies above $2 \mathrm{TeV}$ is increased by a factor of 2-2.3 in the background-dominated region in comparison to the normal operation mode. Thus the required exposure time to measure a spectrum over that energy range is shortened by a factor of $4-5$. Two runs were taken during the night of June 20 at $45^{\circ}$ to $50^{\circ}$ and at $55^{\circ}$ to $60^{\circ}$ zenith angle. For the possible calculation of spectral properties during a high state of the source we have to perform a test on the flux variability between those two 28 minute runs. The observations at $45^{\circ}-40^{\circ}$ and at $55^{\circ}-60^{\circ}$ do have a significant overlap in energy, and a comparison of the flux at, for example, $2 \mathrm{TeV}$ can be done. The $55^{\circ}-60^{\circ}$ data show $49 \pm 8$ events and the $45^{\circ}-40^{\circ}$ data show $14 \pm 4$, which correspond to $35 \pm 10$ events normalized on the same collection area as the $55^{\circ}-60^{\circ}$ zenith-angle observation events. The data at $55^{\circ}$ to $60^{\circ}$ and the run at $45^{\circ}$ to $40^{\circ}$ do show a consistent flux at $2 \mathrm{TeV}$; therefore, no flux variation during these two runs is indicated in the data. Combining those two runs leads to the number of excess events as shown in Table 4 for different energy thresholds.

Table 4 exhibits the statistics that can be achieved in the multi-TeV region using the large zenith-angle technique from a single night of observation with the Whipple Observatory's $10 \mathrm{~m}$ telescope. Most of the statistics beyond $2 \mathrm{TeV}$ are gathered from the $55^{\circ}-60^{\circ}$ zenith-angle range. It should be mentioned that the $\gamma$-ray rate of the flare of June 20 was $1.9 \gamma$-rays minute ${ }^{-1}\left(800 \mathrm{GeV}\right.$ at zenith angle $\left.45^{\circ}\right)$, which is small by comparison with the recently reported burst of $15 \gamma$-rays minute ${ }^{-1}$ above $400 \mathrm{GeV}$ (Gaidos et al. 1996). It is conceivable, therefore, that the large zenith-angle technique can probe energies beyond $20 \mathrm{TeV}$ from Mrk 421 during a strong burst such as that observed by Gaidos et al. (1996).

Data taken on the following night (June 21) indicate a similar flux level of $1.67 \pm 0.32 \gamma$-rays minute ${ }^{-1}$ in the zenith-angle range $45^{\circ}-50^{\circ}$, but a considerable drop to $0.21 \pm 0.20 \gamma$-rays minute ${ }^{-1}$ in the $55^{\circ}-60^{\circ}$ zenith-angle region. If a constant-source spectral index is assumed, this
TABLE 4

Number of EXCESS EVENTS From JunE 20 AT LARge ZeNith ANGLes (MrK 421)

\begin{tabular}{ccc}
\hline \hline $\begin{array}{c}\text { Energy Threshold } \\
(\mathrm{TeV})\end{array}$ & $\begin{array}{c}\text { Excess } \\
\text { Events }\end{array}$ & $\begin{array}{c}\text { Significance } \\
(\sigma)\end{array}$ \\
\hline $0.8 \pm 0.24 \ldots \ldots \ldots$. & 53 & 5.7 \\
$2.0 \pm 0.6 \ldots \ldots \ldots$. & 56 & 6.3 \\
$4.0 \pm 1.2 \ldots \ldots \ldots \ldots$ & 31 & 5.1 \\
$5.0 \pm 1.5 \ldots \ldots \ldots$. & 17 & 4.1 \\
\hline
\end{tabular}

would indicate a significant flux variation on a timescale of $1.5 \mathrm{hr}$ (the time span of the two observations on June 21). This concurs with the reports of flux variability from Mrk 421 on timescales of 30 minutes at lower $\gamma$-ray energies (Gaidos et al. 1996).

\section{SEARCH FOR A SPECTRAL CUTOFF IN THE MULTI-TeV EMISSION FROM MARKARIAN 421}

In previous publications, attempts have been made to derive constraints on the density of intergalactic starlight based on assumed flux absorption at TeV energies. Here, we search for evidence for a spectral cutoff at multi-TeV energies that might be ascribed to such intergalactic absorption. Large zenith-angle data, taken when Mrk 421 is in a high state, are most suitable for searching for such a cutoff.

According to Figures $5 a$ and $5 b$, Mrk 421 was in a high state of emission on June 20,21 , and 28 ( $\geq 1.5 \gamma$-rays minute $\left.{ }^{-1}\right)$. The runs at $45^{\circ}-50^{\circ}$ on all three nights and the run from June 20 at $55^{\circ}-60^{\circ}$ have been used to analyze the $\mathrm{TeV}$ emission as a function of the energy threshold determined by a size cut. Table 5 exhibits the excess events at different energy thresholds combining the data for those three nights. As already indicated by observations on June 20, it is evident (Table 5) from the composite data set (June 20,21, and 28) for the high state that the spectrum of Mrk 421 extends beyond $5 \pm 1.5 \mathrm{TeV}(5.0 \sigma)$. The uncertainty given here is the systematic error in the absolute energy calibration. Although the statistical significance above $8 \pm 2.4 \mathrm{TeV}$ is marginal (3.1 $\sigma$ ), a hint that the emission extends beyond $8 \pm 2.4 \mathrm{TeV}$ during this high state of emission is present in the data. A first spectral analysis shows that there is no obvious sign for a break in the spectrum up to $5 \mathrm{TeV}$ during the high state in 1995 June; however, more detailed studies regarding the energy spectrum are still in progress. At higher energies beyond $8 \pm 2.4 \mathrm{TeV}$, statistics do not support a definitive statement. Figure 6 shows the $\alpha$ distribution for events with energies greater than $5 \pm 1.5$ $\mathrm{TeV}$. It should be noted that the $\alpha$ plot contains very low background contamination and that the signal region $\left(\alpha \leq 15^{\circ}\right)$ may consist entirely of $\gamma$-rays.

TABLE 5

Number of EXCESS EveNTS From JunE 20, 21, aND 28 at Large ZeNith ANgles (MrK 421)

\begin{tabular}{ccc}
\hline \hline $\begin{array}{c}\text { Energy Threshold } \\
(\mathrm{TeV})\end{array}$ & $\begin{array}{c}\text { Excess } \\
\text { Events }\end{array}$ & $\begin{array}{c}\text { Significance } \\
(\sigma)\end{array}$ \\
\hline $0.8 \pm 0.24 \ldots \ldots \ldots$ & 162 & 10.3 \\
$2.0 \pm 0.6 \ldots \ldots \ldots \ldots$ & 109 & 9.3 \\
$4.0 \pm 1.2 \ldots \ldots \ldots \ldots$. & 41 & 6.0 \\
$5.0 \pm 1.5 \ldots \ldots \ldots \ldots$ & 25 & 5.0 \\
$8.0 \pm 2.4 \ldots \ldots \ldots \ldots$ & 10 & 3.1 \\
\hline
\end{tabular}




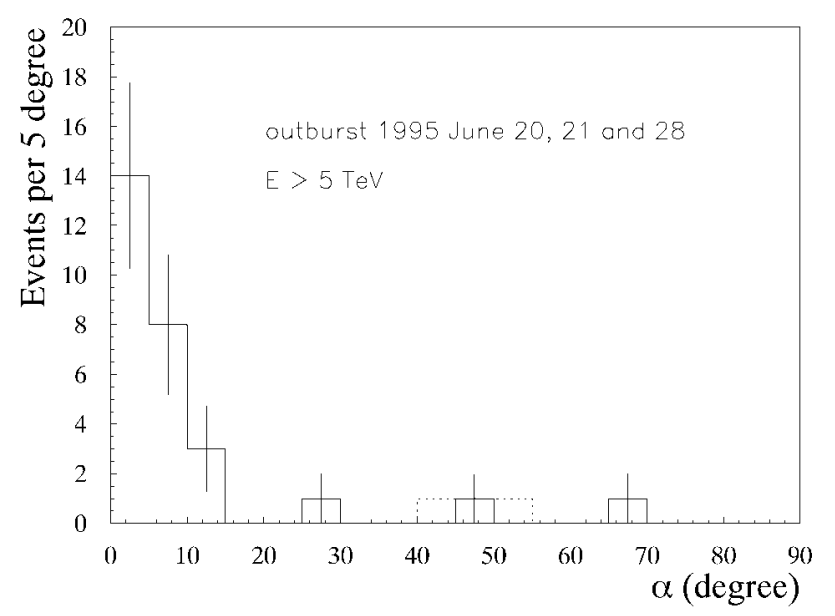

FIG. 6.-The $\alpha$ distribution for the data of June 20, 21, and 28 for a high state of emission at an energy threshold of $5 \pm 1.5 \mathrm{TeV}$. The energy threshold has been defined to ensure that more than $90 \%$ of those events are above $5 \pm 1.5 \mathrm{TeV}$.

\section{DISCUSSION AND SUMMARY}

Probing the near-infrared extragalactic background light (EBL) via its absorption of $\mathrm{TeV}$ photons has been suggested by Gould \& Schrèder (1967). The intrinsic source energy spectra of AGNs in TeV $\gamma$-rays are unknown and therefore any spectral feature or cutoff in the energy spectrum cannot be simply attributed to absorption by the EBL, since it could be an intrinsic source feature as well. First measurements of the energy spectrum of Mrk 421 (Mohanty et al. 1993) showed $\gamma$-rays from Mrk 421 up to energies of $1.5 \pm 0.45 \mathrm{TeV}(3.3 \sigma$ at energies greater than $1.5 \pm 0.45$ TeV). The evidence for $\gamma$-rays above $1.5 \pm 0.45 \mathrm{TeV}$ was statistically marginal. There have been attempts (de Jager, Stecker, \& Salamon 1994) to calculate the EBL density using this data. Those authors interpret the lack of statistics at energies at $5 \mathrm{TeV}$ as the effect of the EBL.

In this work we have specifically searched for multi-TeV emission from Mrk 421 using a new observation and analysis method, the so-called large zenith-angle technique. Monte Carlo simulations suggest that the increase of the detectors' collection area to record $\gamma$-ray-induced showers at zenith angles $45^{\circ}-60^{\circ}$ significantly improves the sensitivity of the Whipple Observatory's $10 \mathrm{~m}$ telescope in the energy region between 1 and $20 \mathrm{TeV}$. Mrk 421 has been observed in 1995 June with this observational technique, and it has been found that the emission of $\mathrm{TeV} \gamma$-rays during a high state seems to extend up to energies greater than $5 \pm 1.5 \mathrm{TeV}$. No apparent evidence for a spectral break can be found up to $5 \pm 1.5 \mathrm{TeV}$, and there is a $3.1 \sigma$ excess above $8 \pm 2.4 \mathrm{TeV}$. However, a more detailed analysis has still to be done to derive the details of the spectrum. As a consequence, there is so far no evidence present in the data for the process of $\left(\gamma \gamma \rightarrow e^{+} e^{-}\right)$interaction of TeV $\gamma$-rays from Mrk 421 with optical or near-infrared photons of intergalactic starlight. But since we do not know the source spectra a priori, we cannot totally exclude the possibility of the presence of the partial absorption of $\mathrm{TeV}$ $\gamma$-rays at $5 \mathrm{TeV}$.

This result is in agreement with the result of Mohanty et al. (1993), since the energy spectrum derived there was not statistically significant beyond $1.5 \pm 0.45 \mathrm{TeV}$, and hence any inference on a cutoff in that spectrum. However, it is important to realize that the data used in this work consist of observations when Mrk 421 was in a high state of emission, comparable to the flux of the Crab Nebula. The data shown by Mohanty et al. (1993) show Mrk 421 in a low state of emission $(0.3 \mathrm{Crab})$. It should be emphasized that the spectrum of Mrk 421 during a high state and a low state are not necessarily expected to agree. However, the conclusions derived from the spectrum of Mohanty et al. (1993) by de Jager, Stecker, \& Salamon (1994), who interpret the data to show a cutoff at $5 \mathrm{TeV}$ by intergalactic starlight, seem to be in contradiction with our new observations. Finally, we want to emphasize the point that using the large zenithangle technique can address the question of the multi-TeV emission of AGNs on short timescales more efficiently than other instruments.

We acknowledge the technical assistance of K. Harris and T. Lappin. This research is supported by grants from the US Department of Energy and by NASA, by PPARC in the UK, and by Forbairt in Ireland.
Biller, S. D., et al. 1995a, ApJ, 445, 227

1995b, Proc. 24th Int. Cosmic-Ray Conf. (Rome), 3, 412

Buckley, J. H., et al. 1996, ApJ, 472, L9-L12

de Jager, O. C., Stecker, F. W., \& Salamon, M. H. 1994, Nature, 369, 294

Gaidos, J. A., et al. 1996, Nature, 383, 319

Gould, R. J., \& Schrèder, G. 1967, Phys. Rev., 155, 1408

Hillas, A. M. 1985, Proc. 19th Int. Cosmic-Ray Conf. (La Jolla), 3, 445

Kerrick, A. D., et al. 1995, ApJ, 438, L59

Kertzman, M., \& Sembroski, G. H. 1994, Nucl. Instrum. Methods Phys. Res., A343, 629

Krennrich, F., et al. 1995, in Towards a Major Atmospheric Cerenkov

Detector, ed. M. Cresti (Padua: Papergraf), 161

\section{REFERENCES}

Lewis, D. A. 1990, Exp. Astr., 1, 213

Mohanty, G., et al. 1993, in Proc. 23rd Int. Cosmic-Ray Conf. (Calgary), 1, 440

1996, in preparation

Quinn, J., et al. 1996, ApJ, 456, L83

Reynolds, P. T., et al. 1993, ApJ, 404, 206

Rose, H. J., et al. 1995, Proc. 24th Int. Cosmic-Ray Conf. (Rome), 3, 464

Sommers, P., \& Elbert, J. W. 1987, J. Phys. G, 13, 553

Tanimori, T., et al. 1994, ApJ, 429, L61

Weekes, T. C. 1994, AIP Conf. Proc. 304, 2nd Compton Symp., ed. C. E. Fichtel, N. Gehrels, \& J. P. Norris (New York: AIP), 270 